УДК 550. 831

\title{
СФЕРЫ ПРИМЕНЕНИЯ СОВРЕМЕННЫХ СТАТИСТИЧЕСКИХ МЕТОДОВ ОБРАБОТКИ ГЕОФИЗИЧЕСКОЙ ИНФОРМАЦИИ
}

\author{
О. М. Муравина ${ }^{1}$ А. С. Долгаль ${ }^{2}$, А. А. Аузин ${ }^{1}$, И. А. Пономаренко ${ }^{1}$, В. Н. Груздев ${ }^{1}$ \\ ${ }^{1}$ Воронежский государственный университет \\ ${ }^{2}$ Горный институт УрО РАН, Пермь
}

Поступила в редакцию 14 октября 2019 г.

\begin{abstract}
Аннотация: в работе рассматриваются результаты применения современного статистического метода обработки геофизических данных - метода группового учета аргументов (МГУА). Приведены примеры использования МГУА при работе с данными геофизических исследований скважин с иелью изучения коллекторских свойств разреза, при статистической обработке результатов наблюдений вариаций естественного электромагнитного поля и в процедуре учета влияния рельефа поверхности намагниченных пород в процессе интерпретации данных аэромагниторазведки. Приведенные примеры практического применения МГУА убедительно свидетельствуют о высокой универсальности данного метода статистической обработки геофизических данныхх.

Ключевые слова: Метод группового учета аргументов, данные геофизических исследований скважин, магнитовариачионные исследования, аэромагниторазведка, учет влияния рельефа.
\end{abstract}

\section{SCOPES OF APPLICATION OF MODERN STATISTICAL METHODS OF PROCESSING OF GEOPHYSICAL INFORMATION}

\author{
O. M. Muravina ${ }^{1}$, A.S. Dolgal ${ }^{2}$, A. A. Auzin ${ }^{1}$, I. A.Ponomarenko ${ }^{1}$, V. N. Grusdev ${ }^{1}$ \\ ${ }^{1}$ Voronezh State University \\ ${ }^{2}$ Geological Mining Institute, Ural Branch of the Russian Academy of Sciences, Perm
}

Received 14 October 2019

\begin{abstract}
The paper considers the results of applying the modern statistical method of processing geophysical data - the method of group accounting of arguments (MGUA). Examples of the use of MGUA when working with logging data to study the reservoir properties of the section, for the statistical processing of the results of observations of variations in the natural magnetic field and in the procedure for taking into account the influence of the relief composed of magnetic rocks when interpreting the data of aeromagnetic exploration are given. The given examples of practical application of MGUA convincingly testify to high universality of this method of statistical processing of geophysical data.

Key words: The method of group accounting of arguments, logging data, magnetovariational studies, aeromagnetic exploration, accounting for the influence of the relief.
\end{abstract}

Введение

Современный уровень развития геоинформационных технологий открывает новые возможности обработки больших объемов геолого-геофизической информации, представленной в цифровой форме. Практическая реализация таких возможностей требует постоянного совершенствования и разработки новых приемов статистического анализа обрабатываемых данных. Методы индуктивного моделирования, к которым относится рассматриваемый метод группового учета аргументов (МГУА), позволяют обеспечить наиболее полное извлечение полезной информации из материалов геолого-геофизических исследований. Как показывают приведенные ниже примеры, МГУА спо-

(C) Muravina O. M., Dolgal A.S., Auzin A. A., Ponomarenko I. A., Grusdev V. N., 2019

Контент доступен под лицензией Creative Commons Attribution 4.0 License.

The content is available under Creative Commons Attribution 4.0 License. 
собен решать широкий круг задач, связанных с обработкой геофизических данных, полученных при проведении исследований на разных уровнях - в скважинах, на земной поверхности и в атмосфере [1].

Метод группового учета аргументов был предложен украинским академиком А. Г. Ивахненко в восьмидесятые годы прошлого столетия [2]. В основу метода положена идея синергетики о возможности существования упорядоченности в сложных системах. Метод позволяет выявить скрытые взаимосвязи между элементами таких систем. С формальной точки зрения МГУА - статистический метод обработки данных, близкий к регрессионному анализу. Однако, в отличие от классического подхода, ни структура регрессионной модели, ни набор конкретных параметров заранее не определяются, а выбираются из множества предложенных в процессе моделирования вариантов. Генерация вариантов моделей осуществляется многорядно, при этом, с переходом в каждый новый ряд, сложность модельного уравнения и набор входящих в него параметров увеличиваются. Оптимальная модель выбирается по минимальным значениям внешних критериев, вычисляемых по данным, не использовавшимся при вычислении коэффициентов модельных уравнений.

Рассмотрим некоторые примеры применения метода, демонстрирующие его перспективность в разных сферах геофизической практики.

\section{Геофизические исследования скважин}

Первые результаты использования МГУА при работе с данными геофизических исследований скважин (ГИС) продемонстрировали возможности и перспективность метода при изучении геологического строения разреза, локализации интервалов оруденения, выявлении областей неоднородностей во вскрытом скважиной разрезе, изучении коллекторских свойств пород, определении характера насыщения коллекторов [3-6]. Положительно оцениваются возможности применения МГУА и для оптимизации комплекса геофизических методов применительно к изучению месторождений рудных полезных ископаемых [7].

С целью совершенствования методики применения МГУА для анализа данных ГИС идентификационное моделирование было выполнено по результатам геофизических исследований 16 скважин на 4 нефтегазовых месторождениях. Общий объем проанализированной информации превысил 100 тысяч точек наблюдений, каждой из которых соответствовал набор данных комплекса ГИС. В результате было получено более 200 модельных уравнений.

Результаты численного эксперимента позволили определить круг задач и предпринять ряд шагов, направленных на модификацию разработанной ранее программы [8] с целью повышения ее эффективности при работе с большими объемами информации. В первую очередь это касалось оптимизации блоков ввода и вывода данных и задания числовых параметров программы. В процессе формирования обучаю- щих и тестовых последовательностей, в качестве исходной информации, помимо собственно данных ГИС, представленных в виде LAS файлов, используются и результаты их геологического истолкования. Выделенные пласты и их геологическое описание сформированы в виде таблиц в формате «dat». Для сведения информации в единую таблицу входных данных разработан блок подготовки, позволяющий автоматизировать процедуру объединения информации и формирования таблицы входных данных для выполнения идентификационного анализа МГУА. Методика подготовки данных состоит из следующих шагов:

1. В программе CurveEditor формируется единый LAS-файл, объединяющий все LAS-файлы, относящиеся к объекту исследования, в котором содержится информация о наблюденных значениях методов ГИС, привязанных к конкретным глубинам. На этом этапе возможно выполнить нормирование данных, которое бывает необходимым если имело место изменение методики проведения исследований (использование различных приборов, разновременные наблюдения и пр.).

2. Единый LAS-файл и интерпретационный файл формата «dat» стандартными средствами MS EXCEL экспортируются в рабочую книгу EXCEL.

3. Программными средствами EXCEL выполняется сопоставление положения кровель и подошв пластов с глубинами точек наблюдений и формируется единая таблица входных данных.

4. Корректировка таблицы. В процессе корректировки исключаются непредставительные данные ГИС, полученные на ограниченных интервалах или неполным комплексом геофизических методов, а также данные, по которым отсутствует геологическая интерпретация.

5. Формализация геологической информации предполагает введение условных числовых идентификаторов моделируемых параметров.

Важной задачей проведенных исследований была отладка параметров работы программы, таких как определение оптимальной длины обучающей и проверочной последовательности наблюдений и выбор наиболее эффективного внешнего критерия.

Длина последовательности наблюдений по одной скважине исчисляется тысячами значений, и оптимизация этого параметра имеет важнейшее значение. Одной из характерных особенностей МГУА является способность выявлять стохастические взаимосвязи по ограниченному количеству исходных данных. Кажется очевидным, что увеличение объема входной информации должно повысить качество идентификационного моделирования. Однако на практике мы столкнулись с тем, что при использовании излишне большого объема имеющихся по скважине данных качество идентификационных моделей ухудшается. Возможно, это связано с увеличением сложности системы и снижением устойчивости решения.

На рис. 1 показана зависимость значений внешнего критерия, по которому оценивается качество моде- 
ли, от длины последовательности наблюдений. Из представленных материалов видно, что при увеличении последовательности наблюдений свыше 700 значений качество моделирования начинает закономерно ухудшаться. Минимумы внешних критериев получены для значений 150 и 400. Исходя из вышесказанного, можно рекомендовать в процессе моделирования постепенное увеличение размера последовательности наблюдений до значений, не превышающих 400-600.

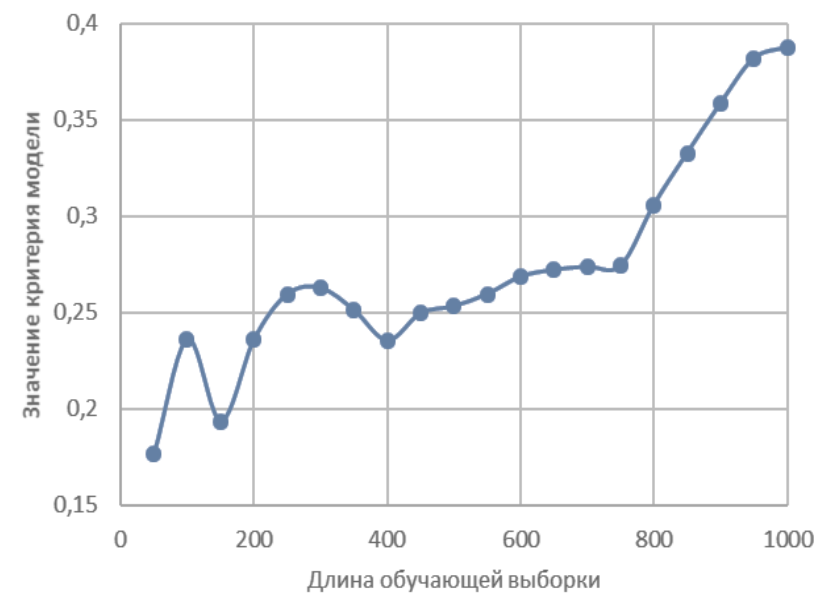

Puc. 1. Зависимость качества модели от длины обучающей выборки.

Усовершенствование процедуры вывода данных было выполнено с целью визуализации результатов моделирование. В настоящее время вывод графиков модельных и экспериментальных данных организован интерактивным образом. Пользователь может обратиться к графическим представлениям результатов и получить информацию о структуре и параметрах каждой модели как для одной, так и для нескольких скважин (рис. 2).

На заключительном этапе был выполнен анализ полученных модельных уравнений. Оказалось что подавляющее большинство лучших моделей, которые описываются соответствующим уравнением [9], получено на 3-м ряду селекции. Количество параметров (данные отдельных методов комплекса ГИС), входящих в модельное уравнение 3 ряда варьирует от 3 до 6 , степень полинома может достигать 4.

Состав методов ГИС, которые вошли в модели в качестве параметров свидетельствует, что наиболее часто в индукционные уравнения входят данные метода самопроизвольной поляризации, результаты измерений удельных электрических сопротивлений потенциал- и градиент-зондами, данные микрозондов и материалы кавернометрии. Реже привлекаются данные индукционного и нейтронного каротажей, а также гамма-каротажа. Эти выводы носят самый общий характер, так как сложность модельных уравнений и набор входящих в него параметров могут существенно изменяться в зависимости от конкретных физико-геологических условий и стоящих перед исследованиями задач.
На рис. 2 в качестве примера приведены графики, построенные по идентификационным уравнениям, полученным на 3-м ряду селекционного отбора. Модельные уравнения имеют вид:

$I K=F(B K, P Z, D S, G Z 4)-$ модель 1 (рис. 2, a)

и $I K=F(P Z, P S, G Z 1, G Z 3)$ - модель 2 (рис. 2, б).

Здесь: $B K$ - боковой каротаж; $P Z$ - каротаж сопротивления потенциал-зондом; $D S$ - кавернометрия; $G Z$ - данные БКЗ (GZ1 - результаты измерений 1-ым градиент-зондом, GZ3 - 3-им градиент-зондом, GZ4 - 4ым градиент-зондом); $I K$ - идентификатор коллектора, который принимает значения: 100 - не коллектор, 200 - водоносный коллектор, 300 - коллектор, содержащий воду и нефть, 400 - нефтеносный коллектор.

Представленные уравнения свидетельствуют, что надежное выделение в разрезе рассматриваемой скважины водоносных коллекторов возможно по результатам измерений удельного электрического сопротивления пород методами бокового каротажа и каротажа сопротивления зондами КС, входящими в набор зондов БКЗ (GZ1,GZ3,GZ4), а также по данным $P S$.

\section{Корреляционный анализ вариаций естественного электромагнитного поля}

Рассмотрим результаты использования МГУА при обработке результатов магнитовариационных исследований, выполненных на территории Воронежского кристаллического массива (ВКМ) в 69 точках, расположенных по неравномерной сети [10]. Метод группового учета аргументов был применен для оценки функциональной зависимости передаточных функций, несущих информацию о глубинной электропроводности разреза.

Идентификационное моделирование было выполнено для пунктов наблюдения, расположенных в различных структурно-тектонических зонах, при различных направлениях поляризации первичного возбуждающего поля и различных периодах $(T)$. При этом, были построены структурно-параметрические идентификационные модели распределения составляющих геомагнитного поля вариаций для периода 1800 с при северо-восточной поляризации первичного поля в зависимости от северного, восточного и северозападного направлений поляризаций первичного поля на периодах 1800 с и 300 с (рис. 3). Выяснилось что, для составляющей $H_{z}$ по всем пунктам наблюдений наиболее тесная взаимосвязь отмечается с вертикальной составляющей при северной (на $T=300$ с) и при восточной (на $T=1800$ с) поляризациях первичного поля. По $H_{\mathrm{x}}$-составляющей поля вариаций практически на всех пунктах наблюдений не отмечается четкой корреляционной зависимости при различных направлениях поляризации поля и периодах. По $H_{y^{-}}$ составляющей по всем пунктам наблюдений отмечается довольно устойчивая корреляция с $H_{\mathrm{x}}$ составляющей на $T=1800$ с при северном и $H_{\mathrm{y}}$ составляющей на $T=1800$ с при восточном направлениях поляризации первичного поля. 


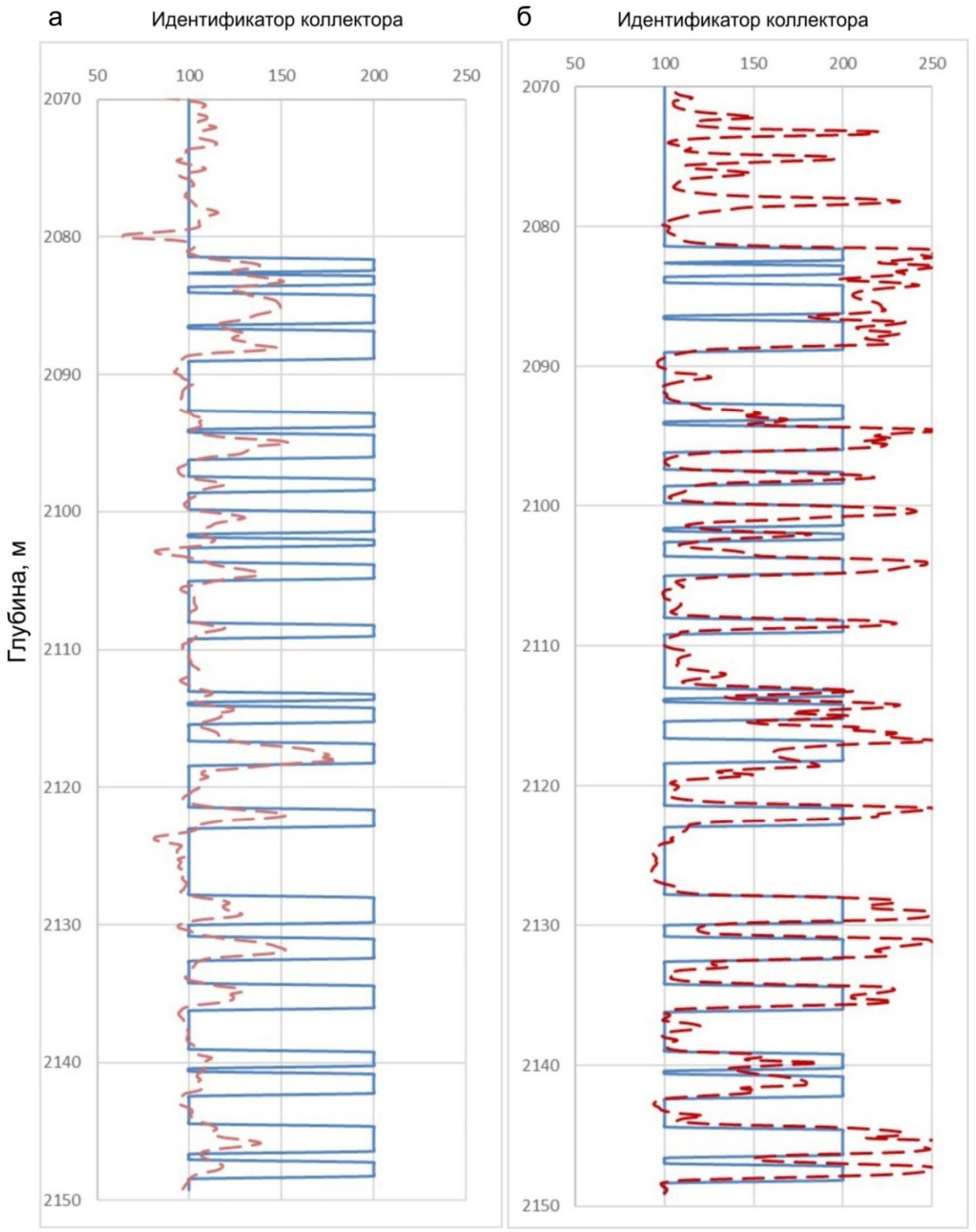

Puc. 2. Результаты идентификационного моделирования: $a$ - модель $1 ; \sigma$ - модель 2 ; сплошной линией показаны экспериментальные значения идентификатора коллектора, пунктиром - модельные значения.

Идентификационное моделирование МГУА подтвердило высокое качество расчета передаточных функций, используемых для анализа синхронной структуры поля по всем трем компонентам на пунктах наблюдения в пределах ВКМ. Анализ корреляционных связей составляющих электромагнитных полей на различных периодах вариаций повышает достоверность реконструкции распределения электромагнитных свойств пород на различных глубинах.

\section{Оценка влияния рельефа местности при проведении аэромагнитных съемок над вулканогенными образованиями}

Примером успешного применения методики МГУА может служить опыт его использования с целью учета влияния рельефа местности при проведении крупномасштабных аэромагнитных съемок. Искажающее влияние рельефа земной поверхности при аэромагнитных наблюдениях является следствием 
влияния двух факторов: аномального эффекта намагниченных объектов, выходящих на дневную поверхность или расположенных в непосредственной близости от нее и «эффекта разновысотности», возникающего из-за различного удаления точек измерения поля от изучаемых магнитовозмущающих объектов за счет изменения высот поверхности наблюдений.
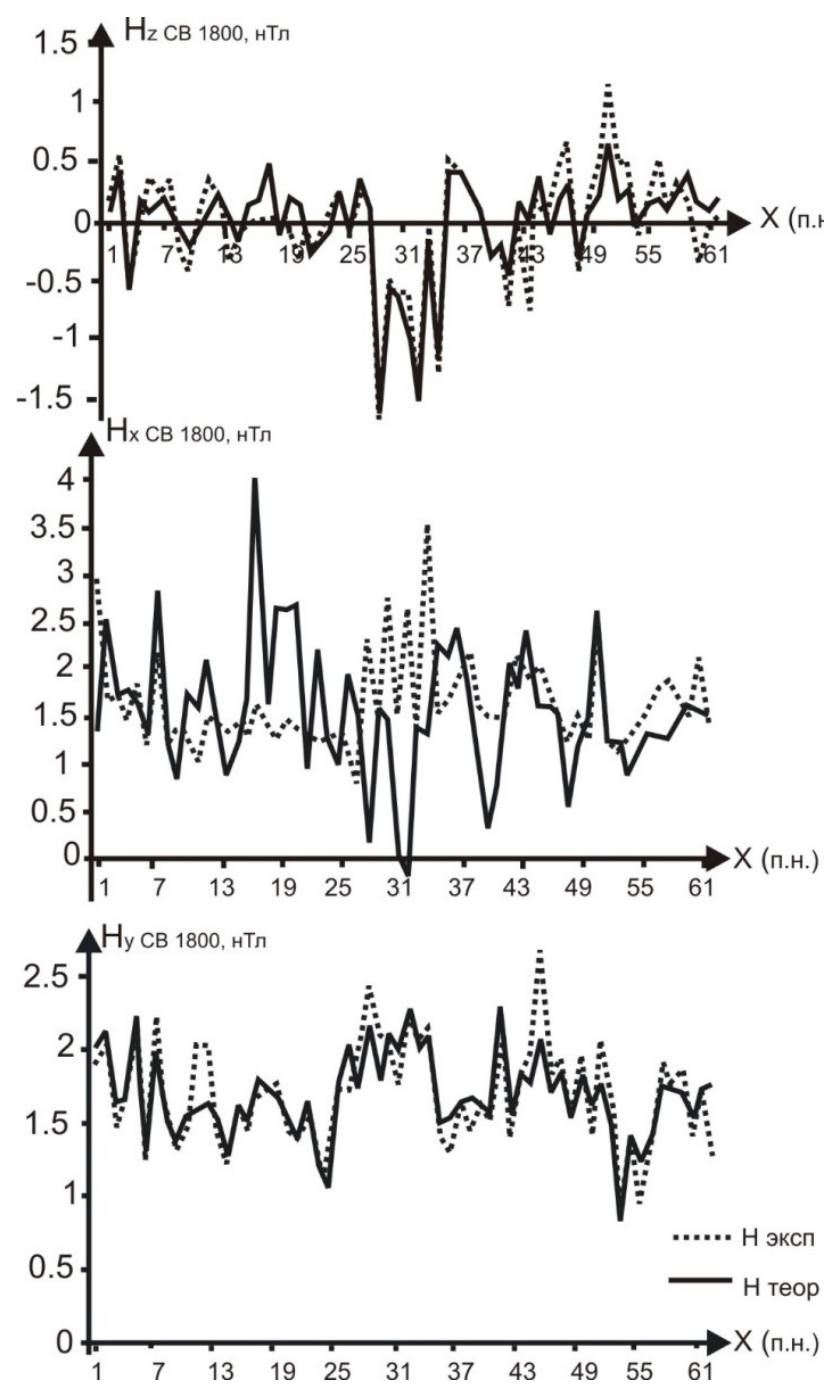

Рис. 3. Идентификационные модели распределения составляющих геомагнитного поля.

Детерминированный подход к вычисление топографических поправок при обработке данных, как магниторазведки, так и гравиразведки, сводится к вычислению аномальных эффектов, создаваемых слагающими рельеф горными породами, при известном пространственном распределении их физических свойств [11]. В случае магниторазведки задача носит существенно более трудоемкий характер. В условиях сильно расчлененного рельефа, при высокой степени латеральной неоднородности магнитных свойств пород верхней части разреза получить приемлемые оценки топографической поправки в рамках упрощенных модельных представлений практически невозможно. Причинами этого являются: дефицит до- стоверной петрофизической информации, неточность задания рельефа вблизи точки расчета, использование модели «плоской» Земли и пр.

Традиционный стохастический способ оценки аномального эффекта рельефа основан на выявлении взаимосвязи между полем $\Delta T$ и высотными отметками рельефом $H$. Однако, в сложных физико-геологических условиях теснота корреляционной линейной взаимосвязи непосредственно между $\Delta T$ и $\mathrm{H}$ может быть очень низкой [12]. Решение может быть более эффективным, если скрытые, возможно нелинейные взаимосвязи между полем и рельефом земной поверхности искать в определенных диапазонах пространственных частот. Эта идея была реализована в 2017 г., когда впервые был предложен способ учета влияния рельефа с использованием метода эмпирической модовой декомпозиции (EMD) в комплексе с МГУА [12, 13]. На первом этапе дискретные значения $\Delta T$ и $H$ разлагаются на эмпирические модовые функции (IMF). Затем МГУА выполняется идентификационный анализ всего набора частотных составляющих магнитного поля и рельефа высот, в результате которого выявляется модель с наиболее тесными взаимосвязями. Метод был протестирован при учете влияния рельефа на результаты аэромагнитной съемки, выполненной НФ ВСЕГЕИ в сложных физико-геологических условиях Норильского района [12]. В результате идентификационного моделирования были установлены составляющие магнитного поля, обусловленные влиянием рельефа ( $\delta T 4, \delta T 5, \delta T 6, \delta T 7)$, а затем рассчитана модель взаимосвязи между суммарной магнитной составляющей $\delta T$ и составляющими рельефа высот $H 4, H 3, H 1$. Модель описывается уравнением, соответствующем 2 ряду селекционного отбора [9]. Качество идентификационных моделей продемонстрировано на рис. 4 .
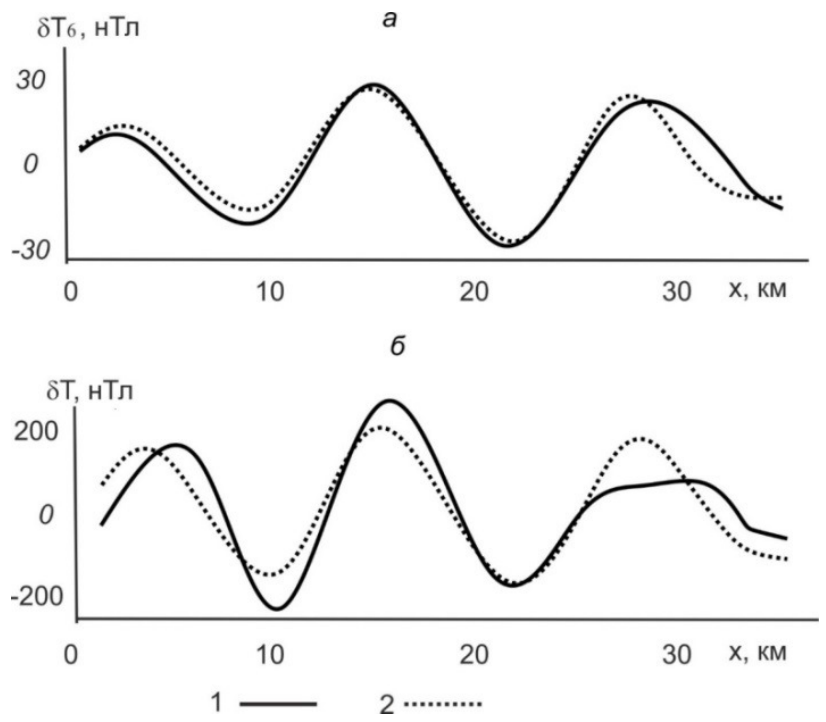

Puc. 4. Результаты идентификационного моделирования низкочастотных составляющих магнитного поля: $a$ - экспериментальные (1) и модельные (2) значения составляющей $\delta T 6$; $\sigma$ - экспериментальные (1) и модельные (2) значения суммарной составляющей $\delta T$. 
Как показали расчеты на практических примерах, предложенный метод определения поправки за рельеф $(\delta \operatorname{Trf})$ согласуются с результатами вычисления топографической поправки решением прямой задачи [14], однако менее трудоемок, не требует априорной информации о магнитных свойствах пород и высотах съемочных полетов и автоматически учитывает сферообразность Земли [15]. Все это открывает широкие перспективы для использования представленного подхода при подготовке геофизической основы для государственных геологических карт масштаба 1:1 $000000-1: 200000$ нового поколения.

\section{Заключение}

Представленные материалы свидетельствуют, что развиваемая авторами современная технология статистического анализа геофизических данных применима к самому широкому спектру геофизических исследований, проводимых не только на земной поверхности, но и в скважинах и воздушном пространстве. Практическая реализация предложенных подходов позволяет повысить достоверность интерпретации геофизических данных и их геологическую информативность.

При анализе многопараметровой комплексной информации использование аппарата МГУА предоставляет возможность оценить вклад каждого из используемых методов в решение стоящих перед исследованиями задач, что может быть положено в основу оптимизации состава комплексов геофизических методов.

Работа выполнена при поддержке грантов РФФИ № 19-05-00654 A, 18-05-00266 A.

\section{ЛИТЕРАТУРА}

1. Никитин, А. А. Теоретические основы обработки геофизической информации: учебное пособие / А. А. Никитин, А. В. Петров. - Москва, Издательство, 2008. -112 с.

2. Ивахненко, А. Г. Индуктивный метод самоорганизации моделей сложных систем / А. Г. Ивахненко. - Киев: Наук. думка, 1982, $296 \mathrm{c.}$

3. Аузин, A. А. Статистический анализ данных каротажа методом группового учета аргументов / А. А. Аузин, О. М. Муравина // Вестник Воронеж. гос. ун-та. Сер.: Геология. 2010. - № 2. - C. 219-224.

4. Муравина, О. М. Метод группового учета аргументов при идентификационном моделировании разрезов скважин / О. М. Муравина // Каротажник. - 2013. - № 6 (228) - С. 32-39.

ФГБУН Горный институт Уральского отделения РАН, Пермь. Долгаль Александр Сергеевич, д. ф.-м. н. E-mail:dolgal@mi-perm.ru; Тел.+7(342)2161008 Воронежский государственный университет Муравина Ольга Михайловна, профессор, д. т. н. E-mail: Comovo@yandex.ru; Тел.: +7 (473)2208385 Аузин Андрей Альбертович, профессор, , д. т. н. E-mail: AAuzin@yandex.ru; Тел.: +7 (473) 2208385 Пономаренко Иван Александрович, аспирант E-mail: kochuma@yandex.ru; Тел.: +7 (473) 2208385 Груздев Владислав Николаевич, доиент, к. ф.-м. н. E-mail: grumerr@rambler.ru; Tел.: +7 (473) 2208385
5. О возможности изучения коллекторских свойств пород методом идентификационного моделирования / О. М. Муравина [и др.] // Каротажник. - 2018. - № 8. - С. 71-80.

6. Muravina, O. M. Possibility of identification of modeling in complex analysis geological and geophysical data / O. M. Muravina, E. I. Davudova, I. A. Ponomarenko // B сборнике: Practical and Theoretical Aspects of Geological Interpretation of Gravitational, Magnetic and Electric Fields Proceedings of the 45th Uspensky International Geophysical Seminar. Cep. "Springer Proceedings in Earth and Environmental Sciences". - 2019. - C. 157-162.

7. Аузин, А. А. О возможности оптимизации комплексов геофизических исследований в скважинах при поисках месторождений рудных полезных ископаемых антеклизы / А. А. Аузин, О. М. Муравина // Вестник Воронеж. гос. унта. Сер.: Геология. - 2013. - № 1. - С. 184-188.

8. Муравина, О. М. Программная реализация метода группового учета аргументов при идентификационном моделировании геолого-геофизических данных / О. М. Муравина, И. А. Пономаренко // Вестник Воронеж. гос. ун-та. Сер.: Геология. -2016 . - №2. - С.107-110.

9. Муравина, О.М. Метод группового учета аргументов при анализе геофизических данных / О. М. Муравина // Геофизика. - 2012. - № 6 - С.10-20.

10. Груздев, В. Н. Коровые аномалии глубинной электропроводности Воронежского кристаллического массива / В. Н. Груздев, В. И. Жаворонкин // Вестник Воронеж. гос. ун-та. Сер.: Геология. - 2015. - №1. - С. 94-101.

11. Ремпель, Г. Г. Актуальные вопросы методики введения поправок, связанных с рельефом местности в данные гравиразведки и магниторазведки / Г. Г. Ремпель // Изв. АН СССР. Сер. Физика Земли. -1980. - № 12. - С. 75-89.

12. Dolgal, $A$. $S$. The reduction of the magnetic field within development areas of the plateaubasalts / A. S. Dolgal, O. M. Muravina, L. A. Hristenko // Geoinformatics 2017, 15-17 May 2017, Kiev, Ukraine. 11143_ENG. Электронная публикация (CD).

13. The empirical mode decomposition and the Hilbert spectrum for nonlinear and non-stationary time series analysis / N.E. Huang [et al.] // Proc. R. Soc. Lond. A. - 1998. - T. 454. - P. 903-995.

14. Долгаль, A. С. Учет влияния рельефа земной поверхности при редуцировании аэромагнитных измерений в пределах больших магматических провинций / А. С/ Долгаль, О. М. Муравина, Н. А. Криволуцкая // Материалы десятых научных чтений памяти Ю. П. Булашевича «Глубинное строение, геодинамика, тепловое поле Земли». - Екатеринбург, 2019 - С. 114-118.

15. Долгаль, А. С. Магниторазведка: компьютерные технологии учета влияния рельефа местности: учеб. пособие / А. С Долгаль // Перм. гос. нац. исслед. ун-т. Пермь, 2014. - 92 с.

Geological Mining Institute, Ural Branch of the Russian Academy of Sciences, Perm. Dolgal A.S., doctor of Physical and Mathematical sciences.E-mail: dolgal@mi-perm.ru Voronezh State University

Muravina O. M., professor, doctor of Technical sciences E-mail: Comovo@yandex.ru; Tel.: +7 (473)2208385 Auzin A. A., professor, doctor of Technical sciences E-mail:AAuzin@yandex.ru; Tel.: +7 (473) 2208385 Ponomarenko I. A., the postgraduate student E-mail: kochuma@yandex.ru; Tel.: +7 (473) 2208385 Grusdev V. N., candidate of Physical and Mathematical sciences, associate professor.E-mail: grumerr@rambler.ru 